\title{
AMENABLE GROUPS AND GROUPS WITH THE FIXED POINT PROPERTY
}

\author{
BY \\ NEIL W. RICKERT $\left(^{1}\right)$
}

Introduction. A left amenable group (i.e., topological group) is a group with a left invariant mean. Roughly speaking this means that the group has a finitely additive set function of total mass 1 , which is invariant under left translation by elements of the group. A precise definition will be given later. Groups of this type have been studied in [2], [5], [7], [8], [12], [13], and [20]. Most of the study to date has been concerned with discrete groups. In this paper we shall attempt to extend to locally compact groups much of what is known for the discrete case.

Another interesting class of groups consists of those groups which have the fixed point property. This is the name given by Furstenberg to groups which have a fixed point every time they act affinely on a compact convex set in a locally convex topological linear space. Day [3] has shown that amenability implies the fixed point property. For discrete groups he has shown the converse. Along with amenable groups, we shall study, in this paper, groups with the fixed point property.

This paper is based on part of the author's Ph.D. dissertation at Yale University. The author wishes to express his thanks to his adviser, F. J. Hahn.

Notation. Group will always mean topological group. For a group $G, G_{0}$ will denote the identity component. Likewise $H_{0}$ will be the identity component of $H$, etc. Banach spaces, topological vector spaces, etc., will always be over the real field. For topology, we use the notation of Kelley [18], except that our spaces will always be assumed Hausdorff.

1. Preliminaries. Let $S$ be an abstract set. We denote by $B(S)$ the Banach space of real valued bounded functions on $S$ with supremum norm.

1.1 Definition. Let $A$ be a closed linear subspace of $B(S)$. A mean on $A$ is a linear functional $m$ such that for each $f$ in $A, m(f) \leqq \sup _{x \in S} f(x)$.

Using the Hahn-Banach theorem, we see that a mean on $A$ can always be extended to $B(S)$. Thus $m(f)$ may be represented as an integral $\int f d m$ for some finitely additive set function $m$ defined on all subsets of $S$. We thus use interchangeably the notations $m(f), \int f d m$ and $\int f(x) d m(x)$. We shall most often be concerned with the case when $S$ is a normal topological space (in fact a locally compact group) and $A$ is the space of continuous bounded functions. In this case

Received by the editors October 13, 1965.

(1) Partially supported by NSF-GP-3509. 
we may consider $m$ to be a regular finitely additive set function defined on the Borel sets. See, for example, Chapter 4 of [6] for the representation theorems for linear functionals.

There is a class of means which are very important. They are the finite means.

1.2 Definition. A mean $m$ on $A$ will be called a finite mean if there are positive scalars $\alpha_{1}, \alpha_{2}, \ldots, \alpha_{n}$ with $\alpha_{1}+\cdots+\alpha_{n}=1$, and points $s_{1}, s_{2}, \ldots, s_{n}$ in $S$, such that for each $f$ in $A$,

$$
m(f)=\alpha_{1} f\left(s_{1}\right)+\alpha_{2} f\left(s_{2}\right)+\cdots+\alpha_{n} f\left(s_{n}\right) .
$$

The important property of the finite means is that they are weak* dense in all means on $A$.

1.3 TheOREM (DAY [2]). Let $A$ be a closed linear subspace of $B(S)$. Then the finite means on $A$ are weak* dense in the set of all means on $A$.

Proof. If not, then a simple application of the separation theorems yields a function $f$ in $A$, such that $m(f) \leqq 1$ for $m$ a finite mean, but $m^{\prime}(f)>1$ contradicting the definition of mean.

We have already given a meaning to the symbol $\int f d m$ for $f$ in $A$, and $m$ a mean on $A$. We wish now to define this integral when $f$ has values in a topological linear space.

1.4 Lemma. Let $S$ be an abstract set, and $A$ a closed linear subspace of $B(S)$. Let $X$ be a compact convex set in a topological linear space $L$, and let $\Phi$ be the family of all linear functionals on $L$ which are continuous on $X$. Suppose that $\Phi$ separates the points of $X$. Let $f$ be a function from $S$ to $X$, such that $\phi \circ f$ is in $A$ for each $\phi$ in $\Phi$. Then if $m$ is a mean on $A$ there is a unique point $x$ in $X$ such that $\phi(x)=m(\phi \circ f)$ for each $\phi \in \Phi$.

Proof (see [3]). Uniqueness is obvious. The existence is clear for finite means, and the general case follows by taking a net of finite means converging to $m$, and taking $x$ to be a limit point of the corresponding points in $X$.

1.5 Definition. Under the hypotheses of the above lemma we shall say that the function $f$ is integrable, and we shall denote the point $x$ by $\int f d m$ or $\int f(s) d m(s)$.

1.6 LEMMA. Let $T$ be an affine transformation of $L$ which is continuous on $X$, and suppose that $A$ contains the constant functions. Then if $f$ is integrable, with values in $X$, Tf is integrable, and

$$
T \int f d m=\int T f d m
$$

Proof. The integrability of $T f$ is easily verified. The equality of $T \int f d m$ and $\int T f d m$ is obvious for finite means, and the general result follows from the fact that the finite means are dense. 
2. Amenable groups. Let $G$ be a group, and $f$ a function defined on $G$. Then by $f_{y}$ we shall mean the function defined by

$$
f_{y}(x)=f(y x)
$$

2.1 Definition. A group $G$ is called "left amenable" if there exists a mean on the bounded continuous real valued functions on $G$ which is invariant under left translations by elements of $G$. That is, $m\left(f_{y}\right)=m(f)$ for $y$ in $G$.

The term left amenable is due to M. Day.

We shall now prove for left amenable groups some basic theorems which are well known if the topology is discrete. Most of them were known to von Neumann [20] for discrete groups.

2.2 TheOREM. A homomorphic image of a left amenable group is left amenable.

Proof. If the homomorphism is denoted by $\pi$ and the invariant mean on $G$ by $m$, then $m^{\prime}(f)=\int f(\pi(x)) d m(x)$ defines an invariant mean on $\pi(G)$.

2.3 Definition. We shall say that a group $G$ is a directed union of subgroups $H_{\alpha}$ if $G$ is the union of the $H_{\alpha}$, and if given $\alpha_{1}$ and $\alpha_{2}$ there is an $\alpha$ such that $H_{\alpha} \supset H_{\alpha_{1}} \cup H_{\alpha_{2}}$.

2.4 THEOREM. If $G$ is a directed union of subgroups $H_{\alpha}$ and if each $H_{\alpha}$ is left amenable, $G$ is left amenable.

Proof. Let $m_{\alpha}$ be an invariant mean on $H_{\alpha}$. Then the map $f \rightarrow \int_{H_{\alpha}} f d m_{\alpha}$ defines a mean on $G$ which is left invariant under $H_{\alpha}$. Denote by $M_{\alpha}$ the set of means on $G$ which are invariant under $H_{\alpha}$. Then the $M_{\alpha}$ are compact sets with the finite intersection property, so they have a nonempty intersection. But any mean in their intersection is an invariant mean on $G$.

2.5 COROLLARY. If the closure of every finitely generated subgroup of $G$ is left amenable, $G$ is left amenable.

2.6 THEOREM. If $H$ is a normal open subgroup of $G$, and if both $H$ and $G / H$ are left amenable, $G$ is left amenable.

Proof. If $\mu$ is a left invariant mean on $H$ and $\mu^{\prime}$ a left invariant mean on $G / H$, observe that if, $f$ is a continuous bounded real valued function on $G, \int f_{y} d \mu$ defines a function on $G$ which is invariant under $H$, and so is constant on cosets of $H$. Thus it defines a function on $G / H$ which is continuous, since our assumptions imply that $G / H$ is discrete. But then

$$
m(f)=\int_{G / H} \int_{H} f(y x) d \mu(x) d \mu^{\prime}(y H)
$$

defines an invariant mean for $G$. 
In the above theorem we required $H$ to be open, in order to guarantee the continuity of $\int f_{y} d \mu$ as a function on $G / H$. There is another important case where this function can be shown to be continuous.

2.7 THEOREM. Let $G$ be either a metric group or a locally compact group. Let $K$ be a compact normal subgroup, and suppose that $G / K$ is left amenable. Then $G$ is left amenable.

Proof. Assume first that $G / K$ is metric. We may construct the mean for $G$ in exactly the same way as in the proof of 2.6 , provided that we can prove that $\int_{K} f(y k) d k$ ( $d k$ is Haar measure) defines a continuous function on the factor group $G / K$. Since $G / K$ is metric, it suffices to prove continuity on sequences. Thus we assume we are given a sequence $y_{n} K \rightarrow y K$ in $G / K$. But it is shown in [23] that the coset representatives $y_{n}$ and $y$ may be so chosen that $y_{n} \rightarrow y$. Then the dominated convergence theorem gives us the desired continuity, since clearly $f_{y_{n}} \rightarrow f_{y}$ (the convergence being pointwise). This completes the proof in the case when $G / K$ is metric. Passing now to the general case, we observe that $G$ is a directed union of its compactly generated open subgroups which contain $K$, so it will suffice to prove that these subgroups are left amenable. Thus suppose that $H$ is a compactly generated open subgroup of $G$, and $H$ contains $K$. Then $H / K$ is open in $G / K$, so if we apply 3.2 (of course we will not use 2.7 when we come to the proof of 3.2) we see that $H / K$ is left amenable. Since $H$ is compactly generated, there is a compact subgroup $K_{0}$ such that $G / K_{0}$ is metric (see [16]). Now $H / K K_{0}$ is metric, and left amenable, being a factor group of $H / K_{0}$ and of $H / K$. Applying the special case we have proved we see that $H$ is left amenable. This completes the proof.

2.8 ExAmples. (i) A compact group is left amenable. The Haar integral provides an invariant mean.

(ii) An abelian group is left amenable. This can be proved for example, by observing that the group acts affinely on the set of means, and applying the MarkovKakutani fixed point theorem. Proofs which depend directly on the Hahn-Banach theorem may also be given. See for example [3], [7], and [20].

(iii) A solvable group is left amenable. It suffices, on account of 2.2 to consider discrete groups. But then the preceding example, together with 2.6, implies the desired result.

(iv) The free group on two generators (with the discrete topology) is not left amenable. See [7] and [20].

3. Subgroups of amenable groups. The principal result of this section is that, if a locally compact group is left amenable, every closed subgroup is also left amenable. This generalizes a theorem of E. Følner for discrete groups. Our proof is based on the idea of Følner's argument, but is necessarily more complicated because of the continuity problems involved. We first have to treat the special case of open subgroups. Here we prove at the same time a similar result for means on uniformly continuous functions, as we shall later need this, and the same proof works. 
3.1 Definition. If $G$ is a topological group, a real valued function $f$ is said to be right uniformly continuous if, given an $\varepsilon>0$, there is a neighborhood $U$ of the identity in $G$ such that

$$
|f(y x)-f(x)|<\varepsilon \text { for all } y \text { in } U, x \text { in } G .
$$

The class of right uniformly continuous functions is easily seen to be a uniformly closed (two-sided) translation invariant linear subspace of $B(S)$.

3.2 TheOREM. If $G$ is left amenable, and if $H$ is an open subgroup of $G, H$ is left amenable.

3.3 THEOREM. If $G$ has a left invariant mean on the space of right uniformly continuous bounded real valued functions, and if $H$ is an open subgroup of $G, H$ also has such an invariant mean.

Proof of 3.2 and 3.3. For each right coset of $H$ pick once and for all a fixed coset representative. Then if $f$ is a function on $H$ we define the function $f^{\prime}$ on $G$ by $f^{\prime}(x)=f(h)$ where $h$ is in $H, x=h g$, and $g$ is one of the chosen coset representatives. Then if $m$ is the invariant mean on $G$ define the invariant mean $m^{\prime}$ on $H$, by

$$
m^{\prime}(f)=m\left(f^{\prime}\right) .
$$

This completes the proof.

We remark that this is exactly the proof used for the discrete case in [8].

We now prove a result which is the basis for eliminating the restriction that $H$ be open in 3.2, provided that $G$ is locally compact.

3.4 TheOREM. Let $G$ be a left amenable group, and $H$ a closed subgroup. Assume that $H \backslash G$, the space of right cosets of $H$, is paracompact, and that local cross sections exist. Then $H$ is left amenable.

Proof. The hypotheses imply that there is a locally finite cover $U_{\alpha}$ of $H \backslash G$, where the sets $U_{\alpha}$ are open, and there is a map $\phi_{\alpha}$ of $U_{\alpha}$ into $G$, such that $\pi \circ \phi_{\alpha}$ is the identity on $U_{\alpha}$, where $\pi$ is the natural map of $G$ onto $H \backslash G$. Let $g_{\alpha}$ be a partition of unity subordinate to the $U_{\alpha}$. If $f$ is a bounded continuous real valued function on $H$, define $f_{\alpha}$ on $\pi^{-1}\left(U_{\alpha}\right)$ by $f_{\alpha}\left(h \phi_{\alpha}(s)\right)=f(h)$ for $h$ in $H$, and $s$ in $U_{\alpha}$. Define $f^{\prime}=\sum_{\alpha} f_{\alpha} g_{\alpha}$. Since the sum is locally finite, $f^{\prime}$ is easily seen to be continuous. Now if $m$ is the invariant mean for $G$, define the invariant mean $m^{\prime}$ for $H$ by

$$
m^{\prime}(f)=m\left(f^{\prime}\right) .
$$

It is not difficult to see that $m^{\prime}$ is indeed an invariant mean for $H$. We omit the details.

3.5 Lemma. Let $G$ be a finite dimensional separable locally compact group. Then if $G$ is left amenable, so is every closed subgroup.

Proof. Karube [17] has shown that in this case $G$ satisfies the hypotheses of 3.4. 
3.6 Lemma. Let $G$ be a separable metric locally compact group. Then if $G$ is left amenable, so is every closed subgroup of $G$.

Proof. The identity component $G_{0}$ of $G$ possesses a largest compact normal subgroup $K$, and $G_{0} / K$ is a Lie group (see [14]). Clearly $K$ is a normal subgroup of $G$, and $G / K$ is finite dimensional. Now suppose that $H$ is a closed subgroup of $G$. Then $H K / K$ is a closed subgroup of $G / K$, so 3.5 shows that $H K / K$ is left amenable, $H /(H \cap K)$ is left amenable. We may now conclude from 2.7 that $H$ is left amenable.

3.7 LemMA. Let $G$ be a locally compact group which is compactly generated. Then if $G$ is left amenable, so is every closed subgroup of $G$.

Proof. We may find a compact normal subgroup $K$ such that $G / K$ is metrizable (see [16] or [19]). Now let $H$ be a closed subgroup of $G$. Then we may apply 3.6 to show that $H K / K$ is left amenable. Then the argument used in the proof of 3.6 may be used to show that $H$ is left amenable.

3.8 LEMMA. Let $G$ be a left amenable locally compact group, and $H$ a compactly generated closed subgroup of $G$. Then $H$ is left amenable.

Proof. We may find a compactly generated open subgroup $M$ of $G$, such that $H$ is a subgroup of $M$. An application of 3.2 shows that $M$ is left amenable, so that 3.7 implies the left amenability of $H$.

3.9 TheOREM. Let $G$ be a left amenable locally compact group. Then every closed subgroup of $G$ is left amenable.

Proof. Let $H$ be a closed subgroup of $G$. Then 3.8 implies that every finitely generated closed subgroup of $H$ is left amenable. An application of 2.4 now completes the proof.

3.10 COROLLARY. A locally compact group is left amenable if and only if the closure of every finitely generated subgroup is left amenable.

Proof. This follows easily from 2.4 and 3.9.

3.11 COROLlary. A left amenable locally compact group does not contain the free group on two generators (the latter group being given the discrete topology).

Proof. This follows from 3.9, since the free group on two generators is not left amenable (see [7]).

4. Groups with the fixed point property.

4.1 Definition. A group $G$ will be said to have the fixed point property, provided that whenever $G$ acts affinely on a compact convex set in a locally convex topological linear space, $G$ has a fixed point. That is, whenever $X$ is a compact convex set in a locally convex space, and $(g, x) \rightarrow g x$ is a continuous map of $G \times X$ to $X$, such that $x \rightarrow g x$ is affine, and such that $\left(g_{1} g_{2}\right) x=g_{1}\left(g_{2} x\right)$, then there is a point $x_{0}$ in $X$ such that $g x_{0}=x_{0}$ for all $g$ in $G$. 
Day has shown that left amenable groups have the fixed point property, and that for discrete groups, the converse is also true. (See [3] and [4].) Slightly modifying his argument we prove:

4.2 THEOREM. A topological group $G$ has the fixed point property if and only if there is a left invariant mean on the space of bounded real valued right uniformly continuous functions on $G$.

Proof. If $m$ is a mean on the bounded right uniformly continuous functions on $G$, we define $g m$ by

$$
g m(f)=m\left(f_{g}\right) \text {. }
$$

Then this is easily seen to define an affine action of $G$ on the compact convex set (with the weak* topology) $M$ of means on right uniformly continuous functions on $G$. But a fixed point in this action is an invariant mean. Suppose now that $G$ has an invariant mean on right uniformly continuous functions. Assume that $G$ acts affinely on a compact convex set $X$ in a locally convex space $L$. Observe firstly that if $\phi$ is a linear functional on $L$ which is continuous on $X$, and if $x_{0}$ is a fixed point of $X$, then $\phi\left(g x_{0}\right)$ is a bounded right uniformly continuous function on $G$ (the uniform continuity may be proved by a standard compactness argument). Thus in the notation of $1.5, g x_{0}$ is an integrable function of $g$. But then 1.6 may be used to show that $\int g x_{0} d m(g)$ is a fixed point under $G$ if $m$ is an invariant mean.

4.3 Remarks. The above proof shows that if $G$ has a fixed point for a certain special action, then it has the fixed point property, this special action being the action on the set of means on right uniformly continuous functions on $G$.

There are two immediate corollaries of 4.2.

4.4 COROLlaRY. Let $G$ have the fixed point property. Then every open subgroup of $G$ has the fixed point property.

Proof. In view of 4.2 this is equivalent to 3.3.

4.5 COROLlaRY. If $G$ is a group and $H$ is a dense subgroup, $G$ has the fixed point property if and only if $H$ has.

Proof. The classes of right uniformly continuous functions on $G$ and $H$ are essentially identical. Thus the result follows from 4.2.

Most of the theorems of $\S 2$ have analogues for groups with the fixed point property.

4.6 THEOREM. If $G$ has the fixed point property so does every homomorphic image of $G$.

4.7 THEOREM. If $G$ is the directed union of subgroups $H_{\alpha}$ and if each $H_{\alpha}$ has the fixed point property, $G$ has the fixed point property.

COROLlaRY. If the closure of every finitely generated subgroup of $G$ has the fixed point property, $G$ has the fixed point property. 
The proofs of these assertions are similar to those of the corresponding theorems in $\$ 2$ if we make use of 4.2 . Alternatively they may be given simple direct proofs based on the definition of the fixed point property.

4.8 THEOREM. Let $G$ be a group, and $H$ a normal subgroup such that both $H$ and $G / H$ have the fixed point property. Then $G$ has the fixed point property.

Proof. Suppose $G$ acts on a compact convex set $X$ in a locally convex space. Then there are points in $X$ fixed under $H$. Thus let $Y$ be the set of fixed points for $H$. It is easily seen that $Y$ is a compact convex set. Furthermore we may deduce from the normality of $H$ (by a simple calculation) that $Y$ is invariant under $G$. Thus there is an induced action of $G / H$ on $Y$, and since $G / H$ has the fixed point property there is a fixed point. But such a fixed point is a fixed point for the action of $G$ on $X$.

We remark that a proof of 4.8 could have been given which used the same method as used in 2.6 (and using 4.2).

4.9 Examples. (i) Abelian groups have the fixed point property. In fact this is the content of the Markov-Kakutani fixed point theorem (see [6]).

(ii) Solvable groups have the fixed point property. This follows from (i) by repeated application of 4.8 .

(iii) Compact groups have the fixed point property. This is the Kakutani fixed point theorem (see [6]), or may be deduced from 4.2 using Haar measure.

(iv) If $G$ is a solvable extension of a compact group (i.e., if there is a solvable closed normal subgroup $S$ of $G$ such that $G / S$ is compact), $G$ has the fixed point property. This follows from (ii) and (iii) together with 4.8 .

(v) An amenable group has the fixed point property. This is an immediate consequence of 4.2 .

(vi) The free group on two generators, with the discrete topology does not have the fixed point property. This follows easily from 4.2 since the free group on two generators is not left amenable.

5. Characterization of groups with the fixed point property. In [9] Furstenberg showed that a connected semisimple Lie group does not have the fixed point property unless it is compact. We give another proof of this theorem. (The theorem is true without connectedness if we require that the identity component is not compact.) Furstenberg then used the theorem to characterize connected Lie groups with the fixed point property. We extend his characterization to locally compact groups $G$ for which $G / G_{0}$ is compact.

5.1 Theorem (FurstenberG). Let $G$ be a semisimple Lie group, the identity component of which is not compact. Then $G$ does not have the fixed point property.

Proof. If $G$ has the fixed point property, so does its identity component, since this is an open subgroup of $G$. Thus we will assume henceforth that $G$ is connected. (We remark that the argument we give would work equally well if $G$ has at most 
finitely many components.) Let $Z$ be the centre of $G$. Then it is well known that $G$ is compact if and only if $G / Z$ is compact (see [10, pp. 123-124]). Thus we may assume that $G$ has a finite centre (since also if $G$ has the fixed point property, so does $G / Z$ ). Thus let $G=K S$ be an Iwasawa decomposition of $G$. (Here $S$ is a closed solvable subgroup, and $K$ a compact subgroup, and every element of $G$ may be written uniquely as $g=k s$.) Then $G$ acts on $G / S$, and thus on the probability measures on $G / S$. If $G$ has the fixed point property it follows that there is a probability measure on $G / S$ which is invariant under $G$. But such a probability measure is known to exist if and only if the modular function of $S$ is the restriction to $S$ of the modular function of $G$. (See [11, pp. 203-207].) But $G$ is unimodular [10, p. 366], while $S$ is not unimodular (the modular function of $S$ is computed explicitly in Chapter 10 of [10], and from the formula given it is easily seen that $S$ is not unimodular). Thus $G$ cannot have the fixed point property.

The restriction that $G$ be a Lie group may now be dropped.

5.2 TheOREM. Let $G$ be a semisimple locally compact group whose identity component is not compact. Then $G$ does not have the fixed point property.

Proof. Denote by $G_{0}$ the identity component of $G$. Let $H$ be an open subgroup of $G$ such that $H / G_{0}$ is compact. Then $H$ has the fixed point property if $G$ does. Now let $K$ be a compact normal subgroup of $H$ such that $H / K$ is a Lie group (which must be semisimple-see [21]). Now if $H$ has the fixed point property, so does $H / K$. Thus the identity component of $H / K$ is compact. Thus $G_{0} K / K$ is compact, so $G_{0} K$ is compact, so $G_{0}$ is compact. That is if $G$ has the fixed point property, $G_{0}$ is compact.

We now characterize locally compact groups $G$ which have the fixed point property, under the assumption that $G / G_{0}$ is compact.

5.3 THEOREM. Let $G$ be a locally compact group, with radical $R$. Then if $G$ has the fixed point property, $G_{0} / R$ is compact. If $G / R$ is compact, $G$ has the fixed point property. Thus if $G / G_{0}$ is compact, $G$ has the fixed point property if and only if $G / R$ is compact.

Proof. The last assertion follows easily from the others. The second assertion is immediate from (iv) of 4.9. The first assertion follows from 5.2, for if $G$ has the fixed point property, so does $G / R$. But $G / R$ is semisimple, so its identity component $G_{0} / R$ is compact.

5.4 COROLlaRY. If a locally compact group $G$ has the fixed point property, so does $G_{0}$.

Proof. Obvious from 5.3.

5.5 THEOREM. Let $G$ be a locally compact group with $G / G_{0}$ compact. Then $G$ has the fixed point property if and only if $G$ is a $(C)$-group, or equivalently, if and only if $G$ does not contain a closed subgroup isomorphic to the free group on two generators. 
Proof. See 5.15 of [21] and apply 5.3 of this paper.

6. Remarks on amenable groups. Since amenable groups have the fixed point property, an immediate consequence of 5.3 is that:

6.1 TheOREM. Let $G$ be a locally compact group with radical $R$. Then if $G$ is left amenable, $G_{0} / R$ is compact.

A certain amount can now be said about giving necessary and sufficient conditions under certain restrictions. It is shown in [22] that for $G / G_{0}$ compact, if $G$ is a $(C)$-group, the regular representation of $G$ weakly contains all unitary representations. But Hulanicki has shown ([12], [13]) that if the regular representation of $G$ weakly contains all representations, $G$ is left amenable. It follows therefore that if $G / R$ is compact, $G$ is left amenable. Thus for groups with $G / G_{0}$ compact, left amenability is equivalent to the fixed point property.

7. Closed subgroups of groups with the fixed point property. If $G / G_{0}$ is compact, and if $G$ has the fixed point property, every closed subgroup of $G$ has the fixed point property, as may be deduced easily from 3.9 together with the remarks in $\$ 6$. We give a different proof here.

7.1 THEOREM. Let $G$ be a locally compact group for which $G / G_{0}$ is compact. Assume that $G$ has the fixed point property. Then every subgroup of $G$ has the fixed point property.

Proof. On account of 4.5 it suffices to consider closed subgroups. Assume firstly that $G$ is a Lie group. Then 5.3 implies that $G / R$ is compact, where $R$ is the radical of $G$. Suppose that $H$ is a closed subgroup of $G$. Then there is a solvable normal subgroup $S$ of $H$, such that $H / S$ is compact. Thus $H$ has the fixed point property. (For the existence of $S$ see 5.4 of [21].) Now consider the general case. Again let $H$ be a closed subgroup of $G$. Choose a compact normal subgroup $K$ of $G$, such that $G / K$ is a Lie group. Then from the special case we have proved we conclude that $H K / K$ has the fixed point property. Thus $H /(H \cap K)$ has the fixed point property, and since $H \cap K$ is compact it has the fixed point property, so $H$ has the fixed point property.

7.2 COROLlaRY. Let $G$ be a locally compact group with the fixed point property. Assume that $G$ has an open normal subgroup $N$ such that $N / G_{0}$ is compact. Then every subgroup of $G$ has the fixed point property. In particular, if $G_{0}$ is open (for example if $G$ is a Lie group) every subgroup of $G$ has the fixed point property.

Proof. The second assertion clearly follows from the first, since we may take for $N$ the identity component. For the first assertion, suppose that $H$ is a subgroup of $G$. Since $N$ is open, $N$ has the fixed point property. Thus $H \cap N$ has the fixed point property. Also $H N$ has the fixed point property, since it is open. Thus $H N / N$ has the fixed point property, so $H /(H \cap N)$ has the fixed point property. But 
since $H \cap N$ has the fixed point property, an application of 4.8 shows that $H$ has the fixed point property.

We sometimes wish to consider subgroups of a group in a topology stronger than the relative topology, and we may wish to know whether 7.1 still holds. (For example, we may be interested in Lie subgroups of a Lie group in the topology which makes the subgroup a Lie group.) Under certain connectedness assumptions 7.1 is valid.

7.3 THEOREM. Let $G$ be a locally compact group with the fixed point property, and let $H$ be a locally compact group such that $H / H_{0}$ is compact. Suppose that there is a one-one (continuous) homomorphism of $H$ into $G$. Then $H$ has the fixed point property.

Proof. Since $H$ has the fixed point property if and only if $H_{0}$ has, we may as well assume that both $G$ and $H$ are connected. But then $G$ is a $(C)$-group by 5.5 . Thus $H$ is a $(C)$-group (see [21]), so 5.5 implies that $H$ has the fixed point property, as asserted.

\section{REFERENCES}

1. M. M. Day, Means for the bounded functions and ergodicity of the bounded representations of semi-groups, Trans. Amer. Math. Soc. 69 (1950), 276-291.

2. — Amenable semi-groups, Illinois J. Math. 1 (1957), 509-544.

3. — Fixed point theorems for compact convex sets, Illinois J. Math. 5 (1961), 585-590.

4. - Correction to my paper "Fixed point theorems for compact convex sets," Illinois J. Math. 8 (1964), 713.

5. J. Dixmier, Les moyennes invariantes dans les semi-groupes et leurs applications, Acta Sci. Math. (Szeged) 12 (1950), 585-590.

6. N. Dunford and J. T. Schwartz, Linear operators. I, Interscience, New York, 1958.

7. E. Følner, A generalisation of a theorem of Bogoliouboff to topological abelian groups with an appendix on Banach mean values in non-abelian groups, Math. Scand. 2 (1954), 5-18.

8. - On groups with full Banach mean value, Math. Scand. 3 (1955), 243-254.

9. H. Furstenberg, A Poisson formula for semi-simple Lie groups, Ann. of Math. 77 (1963), 335-386.

10. S. Helgason, Differential geometry and symmetric spaces, Academic Press, New York, 1962.

11. E. Hewitt and K. A. Ross, Abstract harmonic analysis. I, Springer, Berlin, 1963.

12. A. Hulanicki, Groups whose regular representation weakly contains all irreducible representations, Studia Math. 24 (1964), 37-59.

13. — Amenable topological groups (to appear).

14. K. Iwasawa, On some types of topological groups, Ann. of Math. 50 (1949), 507-557.

15. N. Jacobson, Lie algebras, Interscience, New York, 1962.

16. S. Kakutani and K. Kodaira, Uber das Haarsche Mass in der lokal bikompakten Gruppen, Proc. Imp. Acad. Tokyo 20 (1944), 444-450.

17. T. Karube, On the local cross sections in locally compact groups, J. Math. Soc. Japan 10 (1958), 343-347.

18. J. L. Kelley, General topology, Van Nostrand, Princeton, N. J., 1955.

19. D. Montgomery and L. Zippin, Topological transformation groups, Interscience, New York, 1955. 
20. J. von Neumann, Zur allgemeinen Theorie des Masses, Fund. Math. 13 (1929), 73-116. 21. N. W. Rickert, Some properties of locally compact groups, J. Austral. Math Soc. (to appear).

22. O. Takenouchi, Sur une classe de fonction de type positif sur un groupe localement compact, Math. J. Okayama Univ. 4 (1955), 143-173.

23. $\mathrm{N}$. Th. Varopoulos, $A$ theorem on the continuity of homomorphisms of locally compact groups, Proc. Cambridge Philos. Soc. 60 (1964), 449-463.

YALE UNIVERSTTY,

NeW Haven, Connecticut 